\title{
The influence of rapamycin on the early cardioprotective effect of hypoxic preconditioning on cardiomyocytes
}

\author{
Jiang Wang, YiLiyaer Maimaitili, Hong Zheng, Jin Yu, Hai Guo, Hai-Ping Ma, Chun-ling Chen
}

Department of Anesthesiology, the First Affiliated Hospital of Xinjiang Medical University, Urumqi, Xinjiang, China

Submitted: 21 August 2015

Accepted: 14 December 2015

Arch Med Sci 2017; 13, 4: 947-955

DOI: https://doi.org/10.5114/aoms.2016.59712

Copyright $\odot 2016$ Termedia \& Banach

\section{Abstract}

Introduction: The purpose of this study was to examine the effects of rapamycin on the cardioprotective effect of hypoxic preconditioning (HPC) and on the mammalian target of rapamycin (mTOR)-mediated hypoxia-inducible factor 1 (HIF-1) signaling pathway.

Material and methods: Primary cardiomyocytes were isolated from rat pups and underwent rapamycin and/or HPC, followed by hypoxia/re-oxygenation $(H / R)$ injury. Cell viability and cell injury were determined by 3-(4,5-dimethylthiazol-2-yl)-2,5-diphenyltetrazolium bromide (MTT) and lactate dehydrogenase (LDH) assays, and qRT-PCR was used to measure HIF- $1 \alpha$ and mTOR mRNA expression. A Langendorff heart perfusion model was conducted to observe the effect of rapamycin.

Results: Rapamycin treatment nearly abolished the cardioprotective effect of HPC in cardiomyocytes, reduced cell viability $(p=0.007)$ and increased cell damage $(p=0.032)$. HIF- $1 \alpha$ and mTOR mRNA expression increased in cardiomyocytes undergoing I/R injury within $2 \mathrm{~h}$ after HPC. After rapamycin treatment, mTOR mRNA expression and HPC-induced HIF- $1 \alpha$ mRNA expression were both reduced $(p<0.001)$. A Langendorff heart perfusion model in rat hearts showed that rapamycin greatly attenuated the cardioprotective effect of HPC in terms of heart rate, LVDP, and dp/dtmax (all, $p<0.029$ ).

Conclusions: Rapamycin, through inhibition of mTOR, reduces the elevated HIF- $1 \alpha$ expression at an early stage of HPC, and attenuates the early cardioprotective effect of HPC.

Key words: rapamycin, hypoxic preconditioning, primary neonatal rat cardiomyocytes, hypoxia inducible factor $1 \alpha$.

\section{Introduction}

In heart surgery, ischemic/reperfusion (I/R) occurs after extracorporeal circulation and is a cause of post-operative cardiac dysfunction [1]. Hypoxic preconditioning (HPC), unlike ischemic preconditioning (IPC), may attenuate I/R induced injury to cardiomyocytes [2], and this protective effect has been observed at several hours (early protection lasting several hours) and 12-24 h (late protection lasting several days) after HPC [3]. It is generally accepted that post-transcriptional modification of proteins in the myocardium, and gene transcription and protein synthesis in the myocardium, are involved in HPC-induced cardioprotection. Hypoxia inducible factor 1 (HIF-1) is an important transcriptional factor that is in-

\author{
Corresponding author: \\ Dr. Hong Zheng MD \\ Department of \\ Anesthesiology \\ the First Affiliated Hospital \\ of Xinjiang Medical \\ University \\ Urumqi, China \\ 1 Li Yu Shan St \\ Urumqi, 830011, \\ Xinjiang, China \\ Phone: +8613565996995 \\ E-mail: xjzhenghong@ \\ aliyun.com
}


duced by hypoxic conditions $[4,5]$. Whether HIF-1 is involved in the early cardioprotective effect of HPC is still uncertain [6].

Increasing attention has been paid to the role of rapamycin in cardiovascular disease. However, data on the role of rapamycin with respect to $\mathrm{I} / \mathrm{R}$ injury are inconsistent, or even conflicting. The controversy focuses on whether inhibition of mammalian target of rapamycin (mTOR) after rapamycin treatment inhibits or increases myocardial I/R injury. Raphael et al. [7] reported that the cardioprotective effect of isoflurane preconditioning involves mTOR-mediated HIF-1 signaling pathway activation. In cancer cells mTOR was found to regulate HIF-1 $\alpha$ expression [8]. However, it was later reported that ginsenoside Rg1 could increase HIF- $1 \alpha$ expression in cardiomyocytes, and this effect was attributed to activation of the mTOR receptor [9]. Hernández et al. [10] confirmed that mTOR is activated by I/R and is cardioprotective, that p38-MAPK and Akt are involved in activation of mTOR, and that mTOR inhibition results in hypoxia/reoxygenation-induced cardiomyocyte death. More recent studies, on the other hand, have suggested that inhibition of mTOR with rapamycin protects against reperfusion injury through STAT3 signaling [11, 12].

Based on the aforementioned data, we hypothesize that mTOR may be involved in HPC, and may be affected by the HIF-1 signaling pathway. Thus, the purposes of this study were to examine how rapamycin affects the early cardioprotective effect of HPC, and what effect rapamycin has on the mTOR-mediated HIF-1 signaling pathway.

\section{Material and methods}

\section{Primary cardiomyocytes from rat pups}

Sprague-Dawley (SD) rat pups (1-3 days old, either sex; $n=90$ ) were purchased from the Experimental Animal Center of the First Affiliated Hospital of Xinjiang Medical University. All animals were treated according to the Guide for the Care and Use of Laboratory Animals published by the US National Institutes of Health. This study was approved by the Institutional Review Board of our hospital.

Animals were sterilized with ethanol, fixed on a table, and a thoracotomy was performed. Animals were sacrificed by cervical dislocation. Hearts were harvested while beating and washed with pre-cooled D-Hanks solution (HyClone Laboratories, Inc.) at $4^{\circ} \mathrm{C}$ until the discarded solution was clear. Ten hearts were used for one cell culture, and thus 9 different cultures were performed. The hearts were cut into $1 \mathrm{~mm}^{3}$ blocks and washed with pre-cooled D-Hanks solution 3 times. The heart tissue was transferred to a tube and digested with $0.125 \%$ trypsin (HyClone Laboratories, Inc.; 0.25\% trypsin in PBS containing EDTA) at $37^{\circ} \mathrm{C}$ under continuous shaking. Digestions were performed for 8 , 7,6 , and $6 \mathrm{~min}$. After the first digestion, the suspension was removed. In subsequent digestions, the cell suspension was collected followed by addition of Dulbecco's modified Eagle's medium (DMEM) containing 10\% fetal bovine serum (FBS) (HyClone Laboratories, Inc.) to neutralize the trypsin. After 3 digestions, the supernatant was centrifuged at $1000 \mathrm{rpm}$ for $5 \mathrm{~min}$. The supernatant was collected, and high glucose DMEM containing 10\% FBS was used to maintain the cells.

The cells were seeded into a flask, and incubated with $5 \% \mathrm{CO}_{2}$ at $37^{\circ} \mathrm{C}$ for $1 \mathrm{~h}$ for elimination of adherent fibroblasts. The supernatant was removed, the cells were pipetted, and the cell suspension was added to a 6-well plate followed by incubation with $5 \% \mathrm{CO}_{2}$ at $37^{\circ} \mathrm{C}$ for $48 \mathrm{~h}$, after which the medium was replaced. After incubation for another $24 \mathrm{~h}$, the cardiomyocytes were digested and centrifuged. The cells were grouped and seeded into 96-well plates. For the 3-(4,5-dimethylthiazol-2-yl)-2,5-diphenyltetrazolium bromide (MTT) assay, 6 wells were included in each group $(n=6)$, and for lactate dehydrogenase (LDH) detection 5 wells were included in each group $(n=5)$. A counting plate and trypan blue dye $(0.4 \%)$ were used to determine the cell concentration. The cell density was adjusted to $1 \times 10^{5}$ cells/ well, and then the plates were incubated with $5 \% \mathrm{CO}_{2}$ at $37^{\circ} \mathrm{C}$ for $24 \mathrm{~h}$.

\section{Hypoxia/re-oxygenation (H/R) injury of cardiomyocytes}

Cells were maintained in D-Hanks solution and seeded into a sealed chamber (Modular Chamber, Billups-Rothenberg, Del Mar, CA) that was filled with $95 \% \mathrm{~N}_{2} / 5 \% \mathrm{CO}_{2}$. Ventilation was performed at a rate of $5 \mathrm{l} / \mathrm{min}$ for $15 \mathrm{~min}$, until the concentration of oxygen in the chamber was lower than $1 \%[13,14]$. After incubation at $37^{\circ} \mathrm{C}$ for $2 \mathrm{~h}$, the D-Hanks solution was removed, and high glucose DMEM containing $10 \%$ FBS was added, followed by incubation with $5 \% \mathrm{CO}_{2}$ at $37^{\circ} \mathrm{C}$ for $3 \mathrm{~h}$.

\section{Hypoxic preconditioning (HPC) of cardiomyocytes}

Procedures were identical to those described above for H/R. Hypoxia was maintained for $10 \mathrm{~min}$, and re-oxygenation for $30 \mathrm{~min}$, and this was repeated 3 times. In the HPC $+\mathrm{H} / \mathrm{R}$ group, H/R injury was induced immediately after HPC.

\section{Rapamycin treatment}

Rapamycin (Cayman Chemical Company) was dissolved in DMSO, which was then added to the culture medium. The final concentration of rapa- 
mycin was $20 \mathrm{nM}$ (rapa20). In the control group, only DMSO was added to the medium. Cells were incubated at $37^{\circ} \mathrm{C}$ for $60 \mathrm{~min}$. After incubation, routine cell culture, HPC, and/or H/R injury was performed.

\section{Detection of cell viability by MTT assay}

MTT (MP Biomedicals) was dissolved in PBS at a concentration of $0.5 \mathrm{mg} / \mathrm{ml}$. Following the treatments described above, $20 \mu \mathrm{l}$ of MTT solution was added to each well, followed by incubation at $37^{\circ} \mathrm{C}$ for $4 \mathrm{~h}$. The supernatant was removed, and $150 \mu \mathrm{l}$ of DMSO was added to each well followed by incubation for 20 min to resolve the crystals. The optical density (OD) was measured with a microplate reader at $490 \mathrm{~nm}$. Six wells were included in each experiment, and the average was obtained.

\section{Detection of cell injury by the LDH assay}

An LDH assay was used for detection of cell injury. Following the treatments described above, $0.1 \mathrm{ml}$ of supernatant was collected for the detection of LDH using a kit according to the manufacturer's instructions (Nanjing Jiancheng Biotech Co., Ltd).

\section{Detection of HIF- $1 \alpha$ and mTOR mRNA expression in cardiomyocytes by qRT-PCR}

Primer Premier 5.0 was used to design primers, and $\beta$-actin served as a control. Primers for PCR were synthesized by the Beijing Huada Gene Company. The primers were as follows: HIF-1 $\alpha$ : 5-CAACTGCCACCACTGATGAA-3 (forward), 5-CCACT GTATGCTGATGC CTTA-3 (reverse); mTOR: 5-ATGACGAGACCCAG GCTAAG-3 (forward), 5-GCCAGTCCTCTACAATACGC-3 (reverse). Cells were harvested, and total RNA was extracted with Trizol (Invitrogen). Total RNA was reverse transcribed into cDNA using a kit according to the manufacturer's instructions (Bio-Rad). cDNA was stored until use, and detected after SYBR Green I staining. The threshold cycle (Ct) of HIF- $1 \alpha$, mTOR, and $\beta$-actin was obtained, and a melt curve was employed to determine the specificity of the PCR products. qRT-PCR was used to detect the mRNA expression of HIF- $1 \alpha$, mTOR, and $\beta$-actin, and $\beta$-actin served as an internal reference. The $\Delta \mathrm{Ct}$ was calculated as the difference between the $\mathrm{Ct}$ of the target gene and $\mathrm{Ct}$ of $\beta$-actin. The $2^{-\Delta \Delta \mathrm{ct}}$ method was used to quantify the mRNA expression of the target genes.

\section{Isolated heart perfusion with the Langendorff technique}

Adults rats $(n=48)$ were used for isolated heart perfusion with the Langendorff technique (Langendorff Perfusion System; ADInstruments
Shanghai Trading Co., Ltd). Rats were divided into a control group, HPC group, rapamycin group (Rapa), and Rapa + HPC group ( $n=12$ in each group). The heart rate (HR), left ventricular developed pressure (LVDP), and maximum rate of left ventricular pressure rise $(\mathrm{dp} / \mathrm{dt})$ were recorded.

Animals were fasted for $12 \mathrm{~h}$ before surgery. The rats were intraperitoneally anesthetized with pentobarbital $(60 \mathrm{mg} / \mathrm{kg})$, and heparin (200 U) was administered. Animals were fixed on a table in the supine position, and intratracheal intubation was performed followed by mechanical ventilation. A thoracotomy was performed, and the heart was harvested and placed in Krebs-Henseleit $(\mathrm{K}-\mathrm{H})$ solution $(11.8 \mathrm{mM} \mathrm{NaCl}, 4.7 \mathrm{mM} \mathrm{KCl}$, $12 \mathrm{mM} \mathrm{MgSO}_{4} \cdot 7 \mathrm{H}_{2} \mathrm{O}, 2.5 \mathrm{mM} \mathrm{CaCl}, 24.7 \mathrm{mM}$ $\mathrm{NaHCO}_{3}, 11.0 \mathrm{mM}$ glucose) at $4^{\circ} \mathrm{C}$. The aorta was suspended in the Langendorff perfusion system, and retrograde perfusion was done with $\mathrm{K}-\mathrm{H}$ solution $\left(37^{\circ} \mathrm{C}, \mathrm{pH}=7.37-7.45\right)$ saturated with $95 \% \mathrm{O}_{2}$ and $5 \% \mathrm{CO}_{2}$ at a perfusion pressure of $75 \mathrm{~cm}$ $\mathrm{H}_{2} \mathrm{O}$. During perfusion, the heart was immersed in a constant temperature system $\left(37^{\circ} \mathrm{C}\right)$. After observing a stable heart beat for $30 \mathrm{~min}$ to confirm successful perfusion, a latex bladder was inserted into the left ventricle via the left auricular appendage and connected to a multi-channel signal acquisition system via a pressure transducer. The $\mathrm{HR}$ and LVDP were recorded. Perfusion failure was defined when the HR was < 180 beats/min, left ventricular systolic pressure was $<75 \mathrm{~mm} \mathrm{Hg}$, or coronary flow was $<8 \mathrm{ml} / \mathrm{min}$. The end diastolic LV pressure chosen was $8-10 \mathrm{~mm} \mathrm{Hg}$. Hearts with perfusion failure were excluded from this study.

In the control group, I/R injury was introduced. In brief, ischemia was performed for $30 \mathrm{~min}$, followed by reperfusion for $60 \mathrm{~min}$. HPC was done as follows. The heart was perfused with a hypoxic $\mathrm{K}-\mathrm{H}$ solution for $5 \mathrm{~min}$, and then with an oxygenated $\mathrm{K}-\mathrm{H}$ solution for 5 min. This was performed twice followed by I/R injury. Rapamycin preconditioning was performed as follows. Before HPC or I/R injury, the heart was perfused with a K-H solution containing rapamycin at $0.25 \mathrm{mg} / \mathrm{kg}$ for $15 \mathrm{~min}$. At the end of stable perfusion (T1), after pre-conditioning (T2), after 5 min of reperfusion (T3), after 30 min of reperfusion (T4), and after $60 \mathrm{~min}$ of reperfusion (T5), HR, LVDP, and dp/dt were detected via the signal acquisition system.

\section{Statistical analysis}

Continuous variables are presented as mean \pm standard deviation for each group. The differences between groups were detected by one-way analysis of variance (ANOVA) for time-independent outcomes (such as results of the MTT and $\mathrm{LDH}$ assays and qRT-PCR because all groups were independent) and repeated measures ANOVA for 
time-dependent outcomes (such as results of the Langendorff heart model). Pairwise post-hoc tests using Bonferroni correction were applied when significant findings were found by ANOVA. Statistical analyses were performed with SAS software version 9.2 (SAS Institute Inc., Cary, NC). A 2-tailed $p<0.05$ indicated statistical significance.

\section{Results}

\section{Morphology and viability of primary cardiomyocytes from rat pups}

Under an inverted microscope, the cardiomyocytes immediately after separation were round. Cells showed adherent growth at $4 \mathrm{~h}$, and were completely adherent to the wall and round or spindle-shaped at 19-24 h. Single cell beating was observed occasionally. After $48 \mathrm{~h}$ of culture, cardiomyocytes extended pseudopodia and crosslinks formed between cells into a net-like pattern (a morphological characteristic of surviving cardiomyocytes). Single cell beating at a low frequency was observed, and once crosslinks had formed the frequency of single cell beating increased significantly (about 80-100 beats/minute) (Figure 1).

\section{Early cardioprotection from hypoxic preconditioning}

After hypoxia for $120 \mathrm{~min}$ and re-oxygenation for 180 min, cardiomyocyte injury was induced. In the H/R group, the cardiomyocytes were deformed and shrunken (Figure 2 B) as compared with cells in the control group (Figure $2 \mathrm{~A}$ ). The morphology of cardiomyocytes in the HPC $+\mathrm{H} / \mathrm{R}$ group (Figure $2 \mathrm{C}$ ) was similar to that of cells in the control group.

\section{Cell viability by MTT assay}

Cell viability in each group relative to the control group (defined as $100 \%$ of cells viable) is presented in Figure 3. Rapamycin did not produce a significant effect on cell viability in either the control group (rapamycin: none vs. $20 \mathrm{nM} ; 100.0$ $\pm 6.3 \%$ vs. $99.7 \pm 5.2 \%$, respectively) or the H/R group (rapamycin: none vs. $20 \mathrm{nM} ; 72.0 \pm 4.9 \%$ vs. $73.2 \pm 5.3 \%$, respectively). However, the cell viability in the $\mathrm{HPC}+\mathrm{H} / \mathrm{R}$ group with $20 \mathrm{nM}$ rapamycin was significantly lower than that in the HPC $+\mathrm{H} / \mathrm{R}$ group (72.5 $\pm 6.1 \%$ vs. $84.2 \pm 2.6 \%$, respectively, $p=0.007)$. Cell viability in the $\mathrm{HPC}+\mathrm{H} / \mathrm{R}$ group,
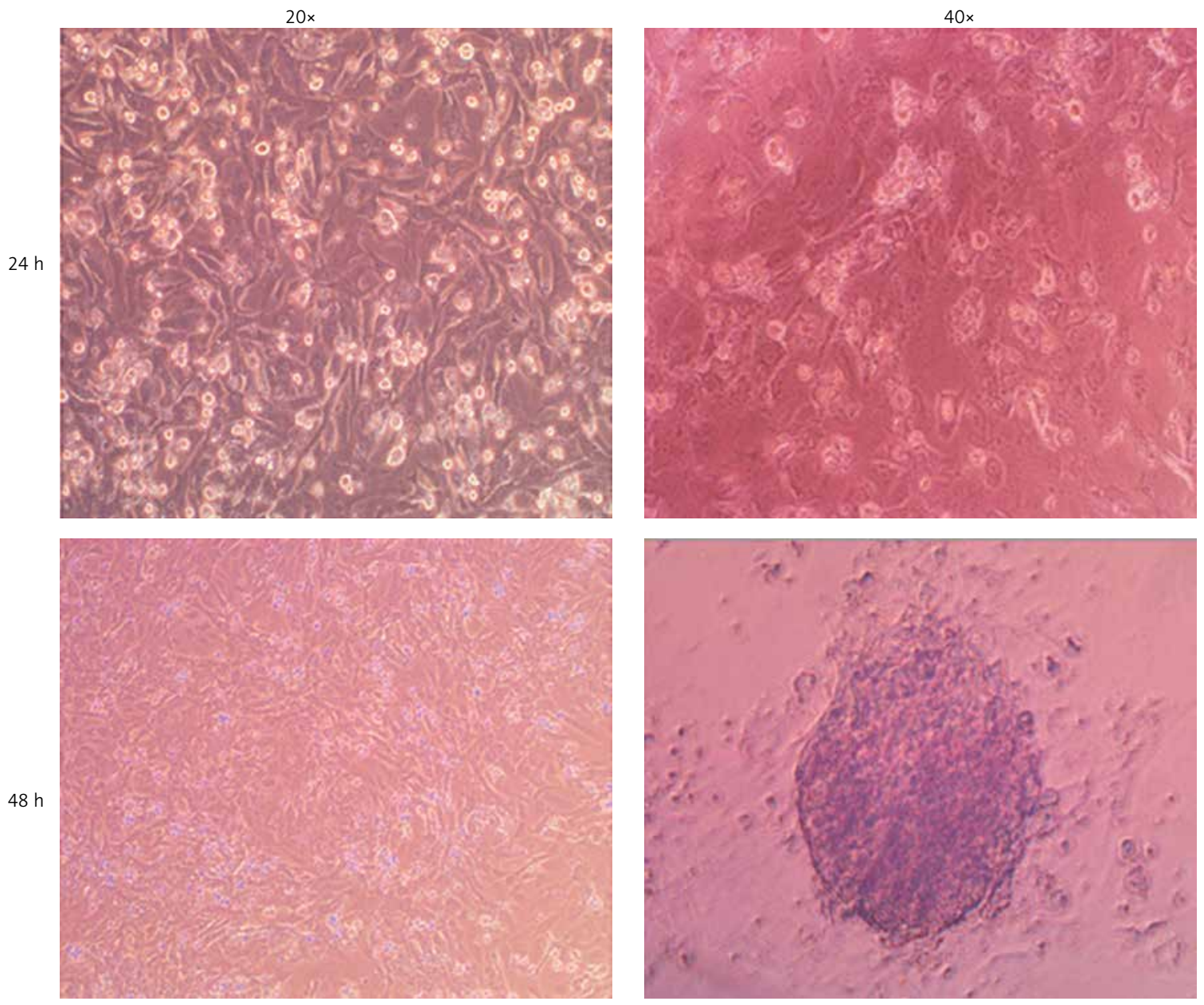

Figure 1. Morphology of primary cardiomyocytes from rat pups at 24 and $48 \mathrm{~h}$ of incubation 

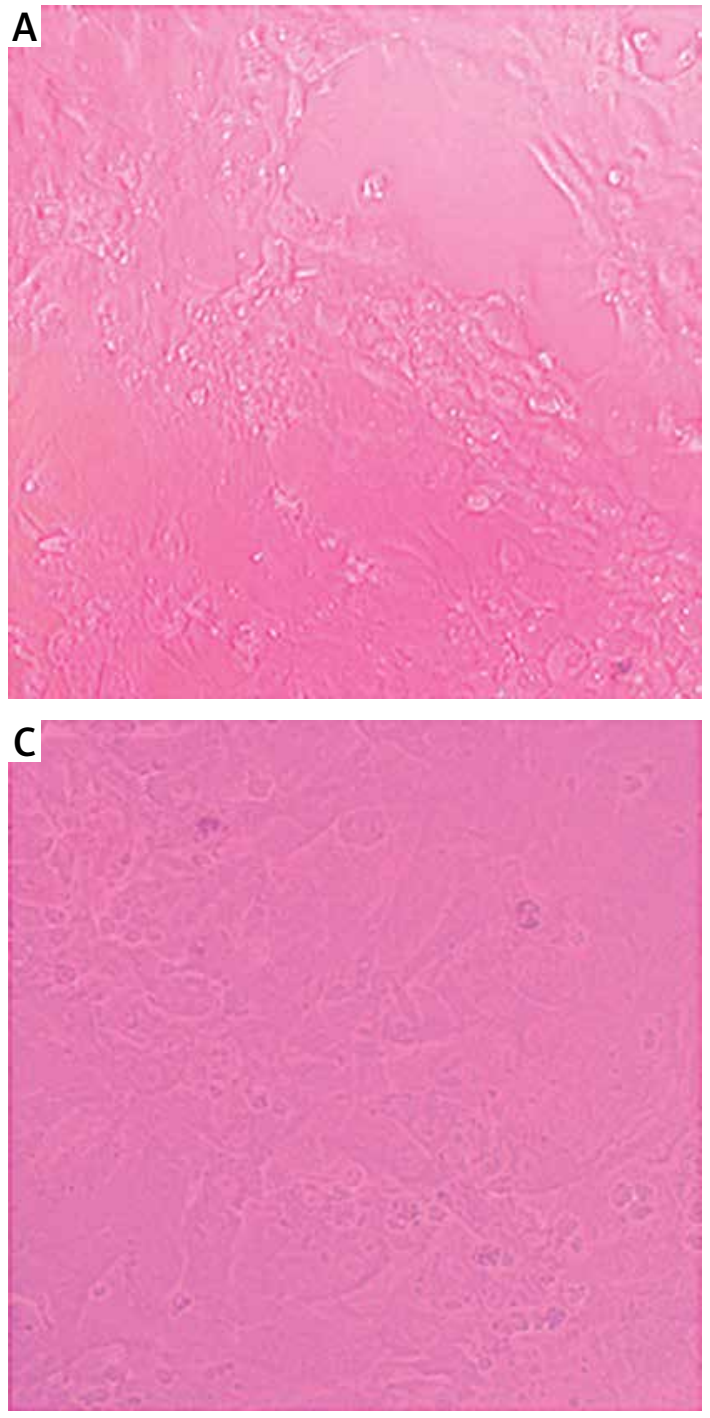

and in the H/R group, was significantly lower than that in the control group (both, $p<0.001$ ), and viability in the HPC $+\mathrm{H} / \mathrm{R}$ group was significantly greater than in the $H / R$ group $(84.2 \pm 2.6 \%$ vs. 72.0 $\pm 4.9 \%$, respectively, $p=0.004)$. Moreover, cell viability in the HPC $+\mathrm{H} / \mathrm{R}+$ rapa20 group and in the $H / R+$ rapa20 group was significantly lower than in the control + rapa20 group (both, $p<0.001$ ). No significant difference in cell viability was found between the HPC $+\mathrm{H} / \mathrm{R}+$ rapa20 group and the $\mathrm{H} / \mathrm{R}+$ rapa20 group.

\section{Damage to cardiomyocytes by LDH assay}

The effect of rapamycin on LDH was only significant in the HPC $+\mathrm{H} / \mathrm{R}$ group $(p=0.032)$ (Table I). $\mathrm{LDH}$ levels in the control group $(p<0.001)$ and the $H P C+H / R$ group were significantly lower than in the H/R group (both, $p<0.001$ ); no significant difference was found between the control and $\mathrm{HPC}+\mathrm{H} / \mathrm{R}$ group. LDH levels in the $\mathrm{HPC}+\mathrm{H} / \mathrm{R}+$ rapa20 group and the $H / R+$ rapa20 group were

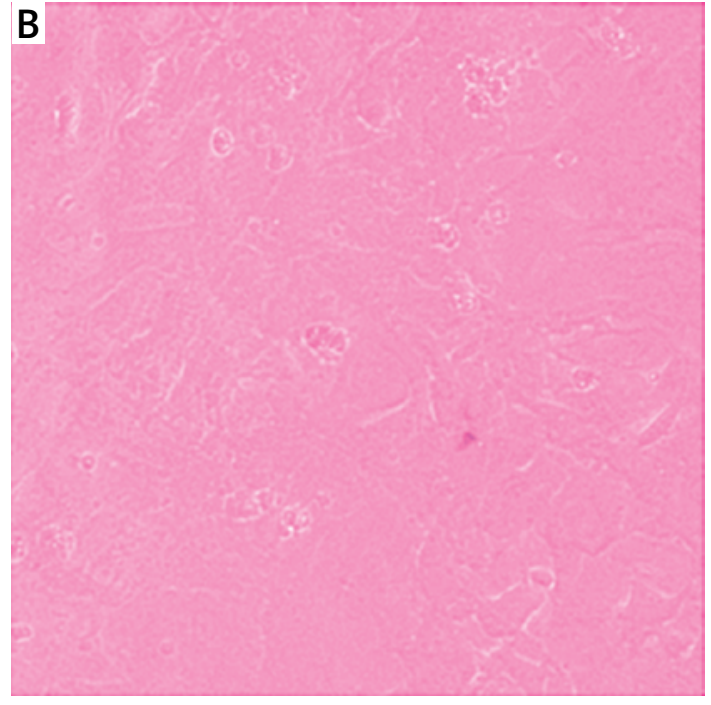

Figure 2. Cells in the hypoxia/re-oxygenation (H/R) group (B) were deformed and shrunken as compared with cells in the control group (A). The morphology of cardiomyocytes in the hypoxic preconditioning (HPC) $+\mathrm{H} / \mathrm{R}$ group $(\mathrm{C})$ was similar to that of cells in the control group (A)

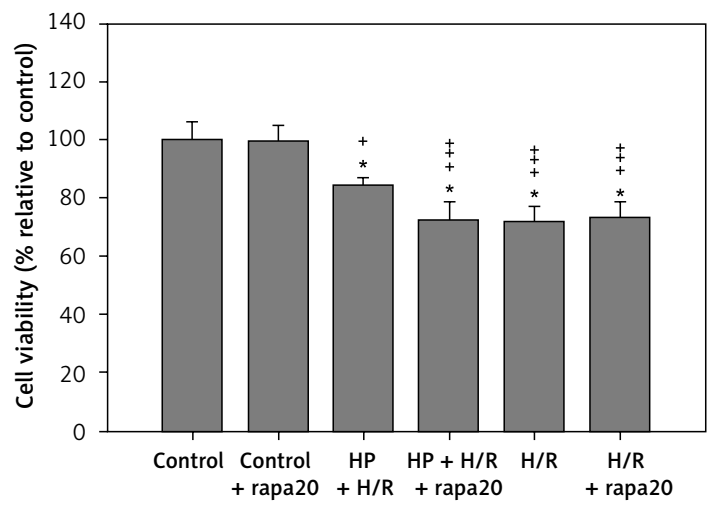

Figure 3. Cell viability measured by MTT assay. Data are presented as mean \pm standard deviation. Six samples were used in each group

${ }^{*}$ Compared to control group, $p<0.05$; ${ }^{\dagger}$ compared to control group with $20 \mathrm{nM}$ rapamycin, $p<0.05$; ${ }^{\ddagger}$ compared to $H P C+H / R$ group, $p<0.05$. P-values were based on a post-hoc test using Bonferroni correction. HPC - hypoxic preconditioning, $H / R$ - hypoxia/re-oxygenation, rapa20 rapamycin $20 \mathrm{nM}$. 
Table I. Cardiomyocyte damage under different conditions as determined by LDH level

\begin{tabular}{|lcccccc|}
\hline Parameter & \multicolumn{2}{c}{ Control } & \multicolumn{2}{c}{ HPC + H/R } & \multicolumn{2}{c|}{ H/R } \\
\hline Rapamycin [nM] & 0 & 20 & 0 & 20 & 0 & 20 \\
\hline LDH [U/l] & 149.67 & 151.16 & 161.61 & 183.33 & 193.04 & 195.53 \\
& \pm 8.74 & \pm 9.61 & \pm 5.65 & $\pm 16.86^{*+\neq}$ & $\pm 8.77^{*+\neq}$ & $\pm 6.04^{*+\neq}$ \\
\hline
\end{tabular}

$H P C$ - hypoxic preconditioning, $H / R$ - hypoxia/re-oxygenation. Data are presented as mean \pm standard deviation. Five samples were used in each group. *Compared to control group, $p<0.05 ;{ }^{\dagger}$ compared to control group with $20 \mathrm{nM}$ rapamycin, $p<0.05 ;{ }^{\ddagger} \mathrm{compared}$ to HPC $+H / R$ group, $p<0.05$. $P$-values were based on a post-hoc test using Bonferroni correction.

significantly higher than in the control + rapa20 group (both, $p<0.001$ ). No significant difference was found between the HPC $+\mathrm{H} / \mathrm{R}+$ rapa20 and the $H / R+$ rapa20 group.

\section{HIF- $1 \alpha$ and mTOR mRNA expression by $\mathrm{qRT}-\mathrm{PCR}$}

The HIF- $1 \alpha$ mRNA expression of the HPC $+\mathrm{H} / \mathrm{R}$ group was significantly greater than that of the $\mathrm{HPC}+\mathrm{H} / \mathrm{R}+$ rapa20 group and the $\mathrm{H} / \mathrm{R}$ group (both, $p<0.001$ ) (Table II). Moreover, mTOR mRNA expression was also significantly higher in the $H P C+H / R$ group as compared to that in the HPC $+\mathrm{H} / \mathrm{R}+$ rapa20 group and the $\mathrm{H} / \mathrm{R}$ group (both, $p<0.001)$. There was no difference in HIF-1 $\alpha$ or mTOR mRNA expression between the HPC $+\mathrm{H} / \mathrm{R}+$ rapa 20 and $H / R$ groups.

Langendorff heart model: heart rate, LVDP, and $\mathrm{dp} / \mathrm{dtmax}$

No animals were excluded; thus, there were 12 in each group. The group effect, time effect, and

Table II. HIF-1 $\alpha$ mRNA expression measured by qRT-PCR

\begin{tabular}{|c|c|c|c|}
\hline $\begin{array}{l}\text { mRNA } \\
\text { expression }\end{array}$ & $H P C+H / R$ & $\begin{array}{c}\mathrm{HPC}+\mathrm{H} / \mathrm{R} \\
\text { + rapa } 20\end{array}$ & $H / R$ \\
\hline HIF- $1 \alpha$ & $2.81 \pm 0.29^{* \dagger}$ & $1.37 \pm 0.24$ & $1.00 \pm 0.06$ \\
\hline mTOR & $2.19 \pm 0.16^{* \dagger}$ & $0.90 \pm 0.08$ & $1.00 \pm 0.07$ \\
\hline \multicolumn{4}{|c|}{$\begin{array}{l}H P C-\text { hypoxic preconditioning, } H / R-\text { hypoxia/re-oxygenation } \\
\text { apa } 20-\text { rapamycin } 20 \mathrm{nM} \text {. Data are presented as mean } \pm \text { standarc } \\
\text { leviation. Three samples were used in each group. }{ }^{*} \text { Compared to } \\
H / R \text { group, } p<0.05 ;{ }^{\dagger} \text { compared to } H P C+H / R+\text { rapa } 20 \text { group } \\
<0.05 . P \text {-values were based on a post-hoc test using Bonferron } \\
\text { orrection. }\end{array}$} \\
\hline
\end{tabular}

the interaction of group and time, were all significant for heart rate, LVDP, and dp/dtmax (all, $p \leq 0.01$ ). These results indicate that the group differences were time-dependent, and should be compared at each time point. No differences in heart rate were noted among the groups until 30 min after reperfusion (Table III). At both $30 \mathrm{~min}$ (T4) and $60 \mathrm{~min}$ (T5) after reperfusion, the heart rate in the HPC groups was significantly higher than in the I/R control group (T4, $p<0.001 ; \mathrm{T}$, $p=0.001$ ). The heart rate in the rapa group (T4 and T5, both, $p<0.001)$ and the rapa + HPC group (T4, $p<0.001 ; \mathrm{T} 5, p=0.029$ ) was significantly lower than in the HPC group, suggesting that HPC did produce a cardioprotective effect. Moreover, the heart rates in the rapa group and the rapa + HPC group were not different from the I/R control group. These results suggest that rapamycin treatment did not exacerbate the decrease in heart rate caused by $I / R$, but it did abolish the cardioprotective effect of HPC.

At both $30 \mathrm{~min}$ (T4) and $60 \mathrm{~min}$ (T5) after reperfusion, LVDP in the HPC group was significantly higher than in the I/R control group (Table IV, both, $p<0.001$ ). The LVDP in the rapa group (T4 and T5, both, $p<0.001)$ and the rapa + HPC group (T4 and T5, both, $p<0.001$ ) was significantly lower than in the HPC group. Moreover, LVDP in the rapa + HPC group was significantly higher than in the rapa group (T4 and T5, both, $p<0.001$ ) and the I/R control group (T4 and T5, both, $p<0.001$ ). No significant difference in LVDP was noted between the rapa group and the I/R control group.

Significant differences in dp/dtmax between the groups were first noted $5 \mathrm{~min}$ into reper-

Table III. Rat heart rates under different experimental conditions using the Langendorff heart model

\begin{tabular}{|lcccc|}
\hline Time points & I/R Control & HPC & Rapa & Rapa + HPC \\
\hline End of perfusion (T1) & $291.17 \pm 10.01$ & $288.58 \pm 13.77$ & $289.25 \pm 10.32$ & $292.08 \pm 12.78$ \\
\hline End of preconditioning (T2) & $291.17 \pm 10.01$ & $282.75 \pm 14.35$ & $290.42 \pm 8.25$ & $291.17 \pm 9.39$ \\
\hline 5 min after reperfusion (T3) & $115.92 \pm 17.43$ & $112.17 \pm 8.23$ & $109.42 \pm 7.99$ & $105.58 \pm 8.05$ \\
\hline 30 min after reperfusion (T4) & $227.58 \pm 20.07$ & $260.00 \pm 14.08^{*}$ & $216.42 \pm 5.84^{\dagger}$ & $228.75 \pm 4.47^{\dagger}$ \\
\hline 60 min after reperfusion (T5) & $259.50 \pm 15.60$ & $279.17 \pm 13.07^{*}$ & $258.83 \pm 9.25^{\dagger}$ & $264.58 \pm 8.88^{\dagger}$ \\
\hline
\end{tabular}

$I / R$ - ischemia/re-perfusion, HPC - hypoxic preconditioning, rapa - rapamycin. All groups received I/R. Data are presented as mean \pm standard deviation. Twelve samples were used in each group. ${ }^{*}$ Compared to I/R control group; ${ }^{\dagger}$ compared to HPC group; $p<0.05$. $P$-values were based on a post-hoc test using Bonferroni correction. 
Table IV. Rat LVDP under different experimental conditions using the Langendorff heart model

\begin{tabular}{|lcccc|}
\hline Time points & I/R Control & HPC & Rapa & Rapa + HPC \\
\hline End of perfusion (T1) & $99.17 \pm 7.31$ & $97.33 \pm 7.41$ & $96.75 \pm 11.62$ & $94.17 \pm 9.00$ \\
\hline End of preconditioning (T2) & $92.33 \pm 9.21$ & $92.42 \pm 7.84$ & $89.33 \pm 8.28$ & $86.75 \pm 7.39$ \\
\hline 5 min after reperfusion (T3) & $8.33 \pm 1.30$ & $8.25 \pm 1.71$ & $7.92 \pm 2.23$ & $8.17 \pm 1.64$ \\
\hline 30 min after reperfusion (T4) & $17.08 \pm 2.75$ & $44.08 \pm 3.29^{*}$ & $17.83 \pm 2.29^{\dagger}$ & $35.17 \pm 4.97^{* \neq}$ \\
\hline 60 min after reperfusion (T5) & $20.92 \pm 3.15$ & $49.92 \pm 4.93^{*}$ & $21.25 \pm 4.58^{\dagger}$ & $28.92 \pm 3.90^{* \neq}$ \\
\hline Recovery ratio & $0.21 \pm 0.03$ & $0.52 \pm 0.06^{*}$ & $0.22 \pm 0.06^{\dagger}$ & $0.31 \pm 0.06^{* \neq}$ \\
\hline
\end{tabular}

LVDP - left ventricular developed pressure, I/R - ischemia/re-perfusion, HPC - hypoxic preconditioning, rapa - rapamycin. All groups received I/R. Recovery ratio $=\angle V D P$ at T5/LVDP at T1. Data are presented as mean \pm standard deviation. Twelve samples were used in each group. *Compared to I/R control group; †compared to HPC group; ${ }^{\ddagger}$ compared to rapa group; $p<0.05$. $p$-values were based on a post-hoc test using Bonferroni correction.

Table V. Rat dp/dtmax under different experimental conditions using the Langendorff heart model

\begin{tabular}{|lcccc|}
\hline Time points & I/R Control & HPC & Rapa & Rapa + HPC \\
\hline End of perfusion (T1) & $3003.25 \pm 289.36$ & $3114.08 \pm 149.74$ & $2943.33 \pm 185.21$ & $2910.92 \pm 201.64$ \\
\hline End of preconditioning (T2) & $2895.92 \pm 227.95$ & $2910.08 \pm 175.10$ & $2860.08 \pm 154.94$ & $2797.50 \pm 169.77$ \\
\hline 5 min after reperfusion (T3) & $606.50 \pm 118.62$ & $865.25 \pm 150.50^{*}$ & $614.50 \pm 115.93^{\dagger}$ & $702.75 \pm 76.27^{\dagger}$ \\
\hline 30 min after reperfusion (T4) & $1042.75 \pm 146.70$ & $2040.00 \pm 249.19^{*}$ & $1070.83 \pm 148.06^{\dagger}$ & $1653.92 \pm 124.21^{* \neq}$ \\
\hline 60 min after reperfusion (T5) & $2001.33 \pm 110.07$ & $2786.33 \pm 152.26^{*}$ & $2019.25 \pm 134.42^{\dagger}$ & $2378.83 \pm 141.65^{*+\neq}$ \\
\hline
\end{tabular}

$I / R$ - ischemia/re-perfusion, HPC - hypoxic preconditioning, rapa - rapamycin. All groups received I/R. Data are presented as mean \pm standard deviation. Twelve samples were used in each group. * Compared to I/R control group; ${ }^{\dagger}$ compared to HPC group; ${ }^{\ddagger}$ compared to rapa group; $p<0.05$. P-values were based on a post-hoc test using Bonferroni correction.

fusion (T3) (Table V). At all 3 time points after reperfusion, dp/dtmax in the HPC groups was significantly higher than in the I/R control group (T3, T4, and T5, all, $p<0.001$ ). The dp/dtmax in the rapa group and the rapa + HPC group was significantly lower than in the HPC group (T3, T4, and T5, all, $p<0.01)$. The dp/dtmax in the rapa + HPC group was significantly higher than in the rapa group (T4 and T5, both, $p<0.001$ ) and the I/R control group (T4 and T5, both, $p<0.001$ ). No significant difference in $\mathrm{dp} / \mathrm{dtm}$ ax was noted between the rapa group and the I/R control group. Therefore, HPC alleviated the reduction in $\mathrm{dp} / \mathrm{dt}$ max starting from the beginning of reperfusion, and rapamycin treatment partially reversed the effect of HPC.

\section{Discussion}

The results of this study showed that HIF-1 $\alpha$ and mTOR participate in the early cardioprotective effect of HPC. Rapamycin inhibits mTOR, which in turn dampens the activation of HIF-1 $\alpha$ expression at an early stage of HPC, and attenuates the early cardioprotective effect of HPC. While there are similar studies in the literature, ours is unique in a number of ways, including the use of the Langendorff heart model and the fact that for the first time rapamycin and HPC were examined together.
Khan et al. [15] reported that rapamycin confers preconditioning-like protection against ischemia-reperfusion injury in isolated mouse heart and cardiomyocytes, which is different than our study. Our study emphasized the role of mTOR in HPC in which rapamycin was used. El-Ani et al. [16] emphasized the cardioprotective effect of rapamycin itself, and the results showed that rapamycin could promote the $\mathrm{Na}^{+} / \mathrm{Ca}^{2+}$ interchange to exert cardioprotection. Our results failed to show that rapamycin alone conferred myocardial protection, which might be ascribed to differences in the dose of rapamycin, time of hypoxia, or severity of hypoxia. Raphael et al. [7] found that rapamycin inhibited HIF-1 $\alpha$ expression to attenuate myocardial protection, similar to the results of our study. However, interventions were different between studies; Raphael et al. used isoflurane for myocardial protection, and in our study HPC was used for myocardial protection. Although intervention with rapamycin alone can confer cardioprotection, rapamycin in combination with isoflurane failed to confer cardioprotection. This is difficult to explain. Our results showed that although HPC was able to confer cardioprotection, its protective effect was abolished by rapamycin, which was related to the inhibition of mTOR and subsequent reduced expression of HIF-1 $\alpha$. These findings were consistent with findings from the study of Raphael et al. 
[7] in which rapamycin was also able to inhibit the cardioprotection of isoflurane.

Our finding that pre-treatment with rapamycin failed to attenuate $\mathrm{H} / \mathrm{R}$ injury in cultured cardiomyocytes is not consistent with those of a prior study [10]. This may be attributed to differences in the methods used for preconditioning. In the study by Hernández et al. [10], H/R was performed for $45 \mathrm{~min}$ and $30 \mathrm{~min}$, respectively. In our study, $\mathrm{H} / \mathrm{R}$ was performed for $2 \mathrm{~h}$ and $3 \mathrm{~h}$, respectively. In addition, autophagy, a protective mechanism that allows cells to eliminate damaged components, is activated by hypoxia [17]. However, different forms of hypoxia can result in alternate regulation of autophagy pathways; in chronic and moderate hypoxia autophagy may be induced through HIF-1 $\alpha$ and PKC $\delta$-JNK1 pathways, whereas in the case of severe and rapid changes in oxygenation autophagy is induced through HIF-independent pathways [17]. Furthermore, autophagy can produce both protective and detrimental effects in cells [17]. Whether the prolonged duration of hypoxia may increase cell injury, and thus compromise the protective effect of rapamycin, requires further study. Interestingly, Möllmann et al. [18] reported that rapamycin elicited a concentration-dependent decrease in fractional cell shortening and in diastolic length in isolated human cardiomyocytes and suggested that the negative inotropic effect might be due to altered calcium homeostasis.

We also found that the mRNA expression of HIF- $1 \alpha$ and mTOR began to increase in cardiomyocytes that underwent H/R injury within $2 \mathrm{~h}$ after HPC. This suggests that HIF- $1 \alpha$ and mTOR mRNA are involved in the initiation of the cardioprotective effect of HPC. After rapamycin preconditioning, mTOR mRNA expression and HPC-induced HIF-1 $\alpha$ mRNA expression were reduced. Based on these findings, and those of prior studies $[8,10$, 19-21], it is possible that mTOR is activated at an early stage of HPC, resulting in increased HIF- $1 \alpha$ expression, and thus a cardioprotective effect. HIF- $1 \alpha$ may serve as an initiator involved in the cardioprotective effect of HPC, and the expression of HIF- $1 \alpha$ may be regulated by rapamycin.

Why the conclusions with respect to the cardioprotective effect of rapamycin vary among studies is still unclear. The differences may be associated with differences in the methodology, animals, and interventions. In addition, the dose of rapamycin, as well as method and timing of rapamycin administration, may also affect cardioprotection. Whether the downstream molecules affected by rapamycin alone are different from those affected by rapamycin used together with other treatments (such as HPC and isoflurane) is still unclear as well. For example, HIF-1 $\alpha$ plays an important role in the cardioprotective effect of iso- flurane and HPC. Rapamycin may inhibit mTOR to reduce HIF- $1 \alpha$ expression, abolishing protection. However, when rapamycin is used alone, cardioprotection is probably independent of HIF- $1 \alpha$, but dependent on mitochondrial KATP channels and the $\mathrm{Na}^{+} / \mathrm{Ca}^{2+}$ exchanger. This might be one reason for the differences among studies.

This study focused on the early stage of HPC, and our findings demonstrated that HPC exerts a protective effect on primary cardiomyocytes at an early stage of preconditioning, and rapamycin could attenuate or even abolish this protective effect. However, the study failed to confirm that single, transient [22], or repeated, long-lasting [14] hypoxic preconditioning exerted a protective effect. This might be attributed to differences in experimental conditions, and the source of the cells. In addition, HIF-1 $\alpha$ expression increased at an early stage of HPC. While infarct size would also be a valid endpoint, it is more predictive of myocardial injury, whereas heart rate, LVDP, and dp/dt are more reflective of a protective effect, which is why they were chosen for this study.

In conclusion, this study demonstrated the involvement of HIF- $1 \alpha$ and mTOR in the early cardioprotective effect of HPC. The early cardioprotective effect of HPC is attenuated in the presence of rapamycin, through the inhibition of $\mathrm{mTOR}$ and the reduced level of HIF- $1 \alpha$ activation in response to HPC. It remains to be determined whether the cardioprotective effect of HPC is related to mTOR activation and subsequent induction of its downstream protective factors, and whether HIF-1 $\alpha$ serves as an initiator to activate endogenous protective factors.

\section{Acknowledgments}

Thank the kind support during this study from the Anesthesiology Department of Xijing Hospital, Xi'an, China.

This study is supported by the National Natural Science Foundation of China (U1303123).

\section{Conflict of interest}

The authors declare no conflict of interest.

\section{References}

1. Lee HT, LaFaro RJ, Reed GE. Pretreatment of human myocardium with adenosine during open heart surgery. J Card Surg 1995; 10: 665-76.

2. Mei DA, Nithipatikom K, Lasley RD, Gross GJ. Myocardial preconditioning produced by ischemia, hypoxia, and a KATP channel opener: effects on interstitial adenosine in dogs. J Mol Cell Cardiol 1998; 30: 1225-36.

3. Nadtochiy SM, Yao H, McBurney MW, et al. SIRT1-mediated acute cardioprotection. Am J Physiol Heart Circ Physiol 2011; 301: H1506-12. 
4. Liang H, Yu F, Tong Z, Yuan B, Wang C. Effect of ischemia post-conditioning on skeletal muscle oxidative injury, mTOR, Bax, Bcl-2 proteins expression, and HIF-1alpha/ beta-actin mRNA, IL-6/beta-actin mRNA and caveolin-3/ beta-actin mRNA expression in ischemia-reperfusion rabbits. Mol Biol Rep 2013; 40: 507-14.

5. Ong SG, Hausenloy DJ. Hypoxia-inducible factor as a therapeutic target for cardioprotection. Pharmacol Ther 2012; 136: 69-81.

6. Sarkar K, Cai Z, Gupta R, et al. Hypoxia-inducible factor 1 transcriptional activity in endothelial cells is required for acute phase cardioprotection induced by ischemic preconditioning. Proc Natl Acad Sci U S A 2012; 109: 10504-9.

7. Raphael J, Zuo Z, Abedat S, Beeri R, Gozal Y. Isoflurane preconditioning decreases myocardial infarction in rabbits via up-regulation of hypoxia inducible factor 1 that is mediated by mammalian target of rapamycin. Anesthesiology 2008; 108: 415-25.

8. Toschi A, Lee E, Gadir N, Ohh M, Foster DA. Differential dependence of hypoxia-inducible factors 1 alpha and 2 alpha on mTORC1 and mTORC2. J Biol Chem 2008; 283: 34495-9.

9. Wang XD, Gu TX, Shi EY, Lu CM, Wang C. Effect and mechanism of panaxoside Rg1 on neovascularization in myocardial infarction rats. Chin J Integr Med 2010; 16: 162-6.

10. Hernández G, Lal H, Fidalgo M, et al. A novel cardioprotective p38-MAPK/mTOR pathway. Exp Cell Res 2011; 317: 2938-49.

11. Das A, Salloum FN, Durrant D, Ockaili R, Kukreja RC. Rapamycin protects against myocardial ischemia-reperfusion injury through JAK2-STAT3 signaling pathway. J Mol Cell Cardiol 2012; 53: 858-69.

12. Das A, Salloum FN, Filippone SM, et al. Inhibition of mammalian target of rapamycin protects against reperfusion injury in diabetic heart through STAT3 signaling. Basic Res Cardiol 2015; 110: 31.

13. Lajoie C, El-Helou V, Proulx C, Clément R, Gosselin H, Calderone $A$. Infarct size is increased in female post-MI rats treated with rapamycin. Can J Physiol Pharmacol 2009; 87: 460-70.

14. Rane S, He M, Sayed D, Yan L, Vatner D, Abdellatif M. An antagonism between the AKT and beta-adrenergic signaling pathways mediated through their reciprocal effects on miR-199a-5p. Cell Signal 2010; 22: 1054-62.

15. Khan S, Salloum F, Das A, Xi L, Vetrovec GW, Kukreja RC. Rapamycin confers preconditioning-like protection against ischemia-reperfusion injury in isolated mouse heart and cardiomyocytes. J Mol Cell Cardiol 2006; 41: 256-64.

16. El-Ani D, Stav H, Guetta V, Arad M, Shainberg A. Rapamycin (sirolimus) protects against hypoxic damage in primary heart cultures via $\mathrm{Na}+/ \mathrm{Ca} 2+$ exchanger activation. Life Sci 2011; 89: 7-14.

17. Fang Y, Tan J, Zhang Q. Signaling pathways and mechanisms of hypoxia-induced autophagy in the animal cells. Cell Biol Int 2015; 39: 891-8.

18. Möllmann H, Nef HM, Kahlert P, et al. Negative inotropic effect of rapamycin on isolated human cardiomyocytes. J Int Med Res 2008; 36: 810-4.

19. Eckle T, Köhler D, Lehmann R, El Kasmi K, Eltzschig HK. Hypoxia-inducible factor-1 is central to cardioprotection: a new paradigm for ischemic preconditioning. Circulation 2008; 118: 166-75.

20. Robador PA, San José G, Rodríguez C, et al. HIF-1-mediated up-regulation of cardiotrophin-1 is involved in the survival response of cardiomyocytes to hypoxia. Cardiovasc Res 2011; 92: 247-55.

21. Kim CH, Cho YS, Chun YS, Park JW, Kim MS. Early expression of myocardial HIF-1alpha in response to mechanical stresses: regulation by stretch-activated channels and the phosphatidylinositol 3-kinase signaling pathway. Circ Res 2002; 90: E25-33.

22. Jiao JD, Garg V, Yang B, Hu K. Novel functional role of heat shock protein 90 in ATP-sensitive $\mathrm{K}+$ channel-mediated hypoxic preconditioning. Cardiovasc Res 2008; 77: 126-33. 